\title{
Dust Formation and the Doubly-Periodic Variable Stars
}

\author{
T. Lloyd Evans \\ South African Astronomical Observatory, P. O. Box 9, Observatory \\ 7935, South Africa
}

\begin{abstract}
The doubly-periodic semiregular variable V Hydrae became redder and developed resonance emission lines and bands during the 199294 deep minimum, which therefore resulted from the production of a circumstellar dust cloud. This removes the need to postulate a secondary pulsation period, ten times as long as the main pulsation period, in the stellar as opposed to circumstellar envelope.
\end{abstract}

\section{Introduction}

RV Tauri variables of the RVb subgroup and many red semiregular variables of type SRb show long secondary periods, which are typically ten times longer than the pulsation period for the semiregular variables and twenty times longer in the case of the RVb stars (Payne-Gaposchkin 1954). Such large ratios imply the presence of a high overtone as well as the fundamental if both periods result from pulsation (Wood 1975). The observation that the doubly-periodic RVb stars have larger near-infrared excesses than the singly-periodic RVa stars suggested that the occurrence of double-periodicity is related to the presence of dust near the star (Lloyd Evans 1985). The less-certain finding that the doubly-periodic semiregular variables of spectral class $M$ have more pronounced silicate emission than other oxygen-rich variables (Lloyd Evans 1987) provided some support for this idea.

V Hydrae, a doubly-periodic ( 530,6670 days) variable which is also a carbon star, is the most spectacular of all these variables with minima which last for several years and are 3.5 mag deep, while the pulsations have an amplitude of 1.2 mag. Spectroscopic observations started in $\mathbf{1 9 8 6}$ and have been continued to date.

\section{Observations}

V Hydrae started to fade early in 1992. Spectra taken then show a unique appearance: the normally steep spectral gradient in the blue became much flatter and the absorption bands of $C_{2}$ strengthened while the emission of $C_{2}$ at 4737 $\AA$, a common feature in carbon-rich Miras and especially marked in V Hydrae in earlier years, disappeared. The blue region became difficult to observe as the carbon star faded because of the greater relative prominence of the blue continuum (Lloyd Evans 1991). Red spectra showed the development of emission lines of the alkali metals, $\mathrm{Na} \mathrm{I,} \mathrm{K} \mathrm{I} \mathrm{and} \mathrm{Rb} \mathrm{I.} \mathrm{Na} \mathrm{I,} \mathrm{for} \mathrm{which} \mathrm{observations} \mathrm{are}$ 
most extensive, showed two emission maxima separated by an interval of several months during which the $\mathrm{D}$ lines were in net absorption although emission was still present. Strong emission of the $4737 \AA$ and $5165 \AA$ bands of $\mathrm{C}_{2}$ appeared and reached maximum strength at the same time as the emission lines. Continuation of the 530 day variation in the $\mathrm{H}$-alpha emission strength suggests that the pulsation continued as normal. JHKL photometry shows that the maximum fading was 2 mag at $J$ and $1 \mathrm{mag}$ at $K$, implying considerable reddening.

The Mira variable $R$ Lep, also a carbon star, underwent a faint cycle in 1994 and showed reddening and spectroscopic phenomena similar to those of $\mathrm{V}$ Hya. R Lep was also observed repeatedly starting in 1986 and this was the first such event, although the spectrum was much more variable from cycle to cycle than in the case of $V$ Hya.

\section{Discussion}

These observations show that the slow variations of $V$ Hydrae, and by implication of other doubly-periodic cool variables, result from absorption by circumstellar matter which appears to have been ejected contemporaneously by the star. The same explanation evidently applies to the occasional faint cycles of Mira variables.

Winters et al. (1994) have made hydrodynamical models of circumstellar dust shells and find that the dust shell may impose a longer-term modulation on the variations of a long period variable. One of their models gives a good representation of the light-curve of $R$ For which had deep minima in 1983 and 1990 (Le Bertre 1992). A new dust layer is formed at each of the deep minima. Fleischer et al. (1992) found that the ejection of a dust shell occurred in every pulsation period if the $\mathrm{C} / \mathrm{O}$ ratio is large but became less frequent if $\mathrm{C} / \mathrm{O}$ is reduced towards one. This is at variance with the systematics of the doublyperiodic semiregular variables for which $P_{2} / P_{1}$ is 9.4 for $\mathrm{M}$ stars but 12.2 for carbon stars (Payne-Gaposchkin 1954). The absence of such well-defined relationships for the Mira variables, which differ from semiregulars primarily in their larger amplitudes, suggests that we do not yet have the complete picture.

\section{References}

Fleischer, A. J., Gauger, A. \& Sedlmayr, E. 1992, A\&A, 266, 321

Le Bertre, T. 1992, A\&AS, 94, 377

Lloyd Evans, T. 1985, MNRAS, 217, 493

Lloyd Evans, T. 1987, in: Circumstellar Matter, A. Appenzeller \& C. Jordan, Dordrecht: Reidel, 541

Lloyd Evans, T. 1991, MNRAS, 248, 479

Payne-Gaposchkin, C. H. 1954, Harvard Annals, 113, 191

Winters, J. M., Fleischer, A. J., Gauger, A. \& Sedlmayr, E. 1994, A\&A, 290, 623

Wood, P. R. 1975, in: Multiple Periodic Variable Stars, W. S. Fitch, Dordrecht: Reidel, 69 